\title{
Nonmotor Symptoms in Early- and Advanced-Stage Parkinson's Disease Patients on Dopaminergic Therapy: How Do They Correlate with Quality of Life?
}

\author{
Peter Valkovic, ${ }^{1,2}$ Jan Harsany, ${ }^{1,3}$ Marta Hanakova, ${ }^{1}$ Jana Martinkova, ${ }^{1}$ and Jan Benetin ${ }^{4}$ \\ ${ }^{1}$ Second Department of Neurology, Faculty of Medicine, Comenius University, Limbova 5, 83305 Bratislava, Slovakia \\ ${ }^{2}$ Laboratory of Motor Control, Institute of Normal and Pathological Physiology, Slovak Academy of Sciences, \\ Sienkiewiczova 1, 81371 Bratislava, Slovakia \\ ${ }^{3}$ Department of Pharmacology and Toxicology, Faculty of Pharmacy, Comenius University, Odbojárov 10, 83232 Bratislava, Slovakia \\ ${ }^{4}$ Department of Neurology, Faculty of Medicine, Slovak Medical University, Ruzinovska 6, 82606 Bratislava, Slovakia
}

Correspondence should be addressed to Peter Valkovic; peter.valkovic@gmail.com

Received 16 January 2014; Accepted 9 February 2014; Published 6 March 2014

Academic Editors: A. Bowirrat and D. T. Dexter

Copyright (C) 2014 Peter Valkovic et al. This is an open access article distributed under the Creative Commons Attribution License, which permits unrestricted use, distribution, and reproduction in any medium, provided the original work is properly cited.

To determine the impact of nonmotor symptoms (NMS) on health-related quality of life (HRQoL) we examined 100 Parkinson's disease (PD) patients on dopaminergic medications. An "early-stage" (ES) and an "advanced-stage" (AS) groups were formed. HRQoL was established by the questionnaire PDQ-8, number of NMS by NMSQuest, and severity and frequency of NMS by the assessment scale NMSS. The total NMS averaged 11.3 (ES = 9.6, AS = 12.8). The NMSS domain correlation profiles for ES and AS did not fundamentally differ; however, the domains attention/memory and mood/apathy correlated moderately to strongly with HRQoL in ES, while the sleep/fatigue domain correlated moderately with HRQoL in AS. Weakly correlating domains were sleep/fatigue in ES and cardiovascular, attention/memory, and mood/apathy domains in AS. In view of these findings we strongly recommend systematic, active screening and therapy for neuropsychiatric disorders (mood, cognitive and sleep disorders, and fatigue) at the initial diagnosis and throughout the entire course of PD.

\section{Introduction}

Parkinson's disease (PD) is a progressive, complex disorder characterized by motor symptoms (bradykinesia, rigidity, resting tremor, and postural instability) as well as a wide range of nonmotor symptoms (NMS) that contribute to significant morbidity and disability $[1,2]$. It is important to identify those NMS that most influence the patient's life, since they may not be evident during the clinical examination [3]. NMS like depression, anxiety, cognitive decline, pain, fatigue, insomnia, and autonomic dysfunction (constipation, urinary symptoms) are significant factors that diminish health-related quality of life (HRQoL) of individuals with PD [4-6]. While common, they are still often underrecognized in clinical practice because patients fail to spontaneously mention them, and healthcare professionals also fail to systematically ask about them $[5,7]$.
Nonmotor symptoms occur not only in advanced but also in early stages of PD. Some symptoms, for example, olfactory deficit, constipation, rapid-eye movement sleep behavior disorder, and depression, can even precede the appearance of motor symptoms by many years [8]. Most of the PD-associated NMS are believed to be caused by the nondopaminergic systems, in accordance with the hypothesis that other neurotransmitters including serotoninergic, noradrenergic, and cholinergic transmission are involved [9]. However, in a recent study on advanced PD subjects, Storch and coworkers [10] showed that seven out of ten selected NMS (with the exception of dysphagia, excessive sweating, and bladder urgency) fluctuated in conjunction with motor symptom fluctuations. Moreover, they were also more frequent and more severe in "off" than in the "on" state. This evidence indicates that any optimization of dopaminergic therapies must also address the management of NMS. 
In light of the above facts, our study aimed to determine the impact of NMS on HRQoL in a cohort of Central European PD patients from the Slovak Republic who were already on dopaminergic medications. We hypothesized that the involvement of individual NMS domains would differ depending on the disease stage. Therefore, special emphasis was placed on a comparison of early-stage and advancedstaged PD patients.

\section{Subjects and Methods}

2.1. Subjects. One hundred consecutive patients with idiopathic PD diagnosed according to the UK Parkinson's Disease Society Brain Bank criteria [11] were recruited from two specialized movement disorder units of tertiary centers. All subjects gave their informed written consent in accordance with the Declaration of Helsinki, and the local Ethics Committee approved the study protocol. Patients with mild cognitive impairment were included if their informed written consent could be obtained and they were able to complete clinimetric questionnaires alone or with the help of their caregiver. All patients had been on dopaminergic therapy with levodopa (plus dopa decarboxylase inhibitor) and/or dopamine agonists for at least 3 months. Untreated subjects or those on advanced therapies like deep brain stimulation, subcutaneous apomorphine pump, or intrajejunal levodopa infusion pump were not included. For analysis the patients were divided into "early-stage" (ES) and "advanced-stage" (AS) groups on the basis of the modified Hoehn and Yahr score (H\&Y) [12], the cut-off of 2.5, and a history of late complications of levodopa therapy. Six patients were classified in H\&Y1, 1 in H\&Y1.5, 31 in H\&Y2, and 9 in H\&Y2.5 (ES subjects). Forty-five people were in H\&Y stage 3, 7 in H\&Y4, and 1 in H\&Y5 (AS patients). Dopaminergic therapy load was expressed in terms of levodopa equivalent daily dose (LEDD) [13]. Each person was in the state in which Parkinson motor symptoms were best controlled during assessment. Fluctuating patients were assessed in their optimal "on" state. Demographic and basic clinical data are shown in Table 1.

2.2. Methods. Validated Slovak translations of the following screening and diagnostic instruments were applied:

(i) the Parkinson's disease questionnaire with eight dimensions (PDQ-8), health-related quality of life measure [14], a summary index (PDQ-8 SI) which was used for statistical analysis,

(ii) the nonmotor symptoms screening questionnaire (NMSQuest), a 30-item tool, which records the presence or absence of nonmotor symptoms [15],

(iii) the nonmotor symptom assessment scale for Parkinson's disease (NMSS), a 30-item scale for assessing NMS, which covers nine dimensions: cardiovascular, sleep/fatigue, mood/apathy, perceptual problems, attention/memory, gastrointestinal, urinary, sexual function, and miscellaneous [16].
Questionnaires PDQ-8 and NMSQuest were completed by patients (with the aid of caregivers if necessary) while waiting to be seen by a neurologist. The NMSS was administered by a trained investigator.

2.2.1. Statistical Analysis. Statistical examination of the data was performed using IBM SPSS Statistics 20. Clinical and demographic variables of both groups of PD patients were compared by Student's $t$-test $(P<0.05)$. The KolmogorovSmirnov test showed that not all data were normally distributed, so Spearman's rank correlation coefficient (Rho) was used to evaluate the association between PDQ-8 and demographic and clinical variables, NMSQuest score, NMSS total score, and nine different NMSS domains. The strength of the association for correlation coefficients was interpreted as follows: $\leq 0.19$, negligible; 0.20 to 0.39 , weak; 0.40 to 0.59 , moderate; 0.60 to 0.79 , strong; and $\geq 0.80$, very strong [17].

\section{Results}

Compared to ES patients, AS subjects had significantly higher scores on the PDQ-8, NMS-Quest (number of NMS), and NMSS (Table 1). The average declared number of NMS in all 100 patients was 11.3; the average number in ES was 9.6, and in AS 12.8.

Table 2 presents the correlations found between quality of life measure PDQ-8 and selected variables of interest. The evaluation of all 100 patients showed a weak correlation with the duration of PD and NMSS cardiovascular aspects, NMSS sleep/fatigue, NMSS/perceptual problems, NMSS/gastrointestinal aspects, and NMSS/miscellaneous. The H\&Y score, NMSQuest, NMSS total, NMSS mood/ apathy, and NMSS attention/memory domain revealed correlations, which were moderate to strong. Similar moderateto-strong relationships were seen in ES subjects. Moreover, there was a weak correlation with NMSS sleep/fatigue. The AS group had a PDQ-8 score that correlated moderately with NMSQuest, NMSS total, and NMSS sleep/fatigue scores. Weak correlations were found for NMSS cardiovascular, NMSS mood/apathy, and NMSS attention/memory domains.

\section{Discussion}

Although NMS greatly influence the HRQoL of PD patients, more than $50 \%$ of existing NMS are not identified in clinical practice [5].

The prevalence of overall NMS in our cohort of 100 PD subjects on dopaminergic drugs was $11 / 30$. This is as high as reported by most other studies. [3, 5, 15, 16, 18] According to the NMSQuest (evaluation of prevalence) and NMSS (frequency $\times$ severity) the nonmotor symptoms were significantly associated with poor HRQoL across the entire clinical spectrum of PD. This finding agrees with the proposal to consider the frequency and severity of nonmotor symptoms as a whole to be the most important predictor of HRQoL in PD [3].

Surprisingly, separate evaluation of NMSS domain correlation profiles for early-stage and advanced-stage groups did 
TABLE 1: Comparative statistics of demographic and basic clinical data and of scores of selected screening and diagnostic instruments.

\begin{tabular}{|c|c|c|c|c|}
\hline & All & ES & AS & $P$ value of $t$-test ${ }^{*}$ \\
\hline$N$ & 100 & 47 & 53 & \\
\hline Gender & $50 \mathrm{M}: 50 \mathrm{~F}$ & $26 \mathrm{M}: 21 \mathrm{~F}$ & $24 \mathrm{M}: 29 \mathrm{~F}$ & \\
\hline Hoehn and Yahr score & $2.6 \pm 0.7(1-5)$ & $2.0 \pm 0.4(1-2.5)$ & $3.2 \pm 0.4(3-5)$ & $<0.01$ \\
\hline Age $( \pm S D) ;$ years & $65.5 \pm 8.8(47-85)$ & $62.9 \pm 8.0(47-76)$ & $67.9 \pm 8.9(47-85)$ & $<0.01$ \\
\hline Disease duration; years & $5.9 \pm 4.4(0.5-19)$ & $4.1 \pm 3.4(0.5-14)$ & $7.5 \pm 4.6(0.5-19)$ & $<0.01$ \\
\hline LEDD; mg & $935.5 \pm 476.6(100-2000)$ & $702.1 \pm 337.0(100-1500)$ & $1142.5 \pm 489.1(300-2000)$ & $<0.01$ \\
\hline NMSQuest score $^{O}$ & $11.3 \pm 4.6(0-20)$ & $9.6 \pm 4.4(0-18)$ & $12.8 \pm 4.4(1-20)$ & $<0.01$ \\
\hline NMSS total score ${ }^{\circ}$ & $44.2 \pm 26.7(1-170)$ & $37.7 \pm 20.5(1-82)$ & $50 \pm 30.2(11-170)$ & $=0.02$ \\
\hline PDQ-8 summary index ${ }^{\circ}$ & $29.3 \pm 17.8(0-69)$ & $21.9 \pm 15.0(0-53)$ & $35.8 \pm 17.6(0-69)$ & $<0.01$ \\
\hline
\end{tabular}

Abbreviations: AS: advanced-stage Parkinson's disease; ES: early-stage Parkinson's disease; M: male; F: female; LEDD: levodopa equivalent daily dose.

${ }^{*}$ Comparison between early-stage and advanced-stage patients.

${ }^{\circ}$ For explanation, see Section 2.2.

Values are displayed as arithmetic mean \pm standard deviation (range).

TABLE 2: Correlations expressed as Spearman's rank correlation coefficient between quality of life measure and selected variables of interest.

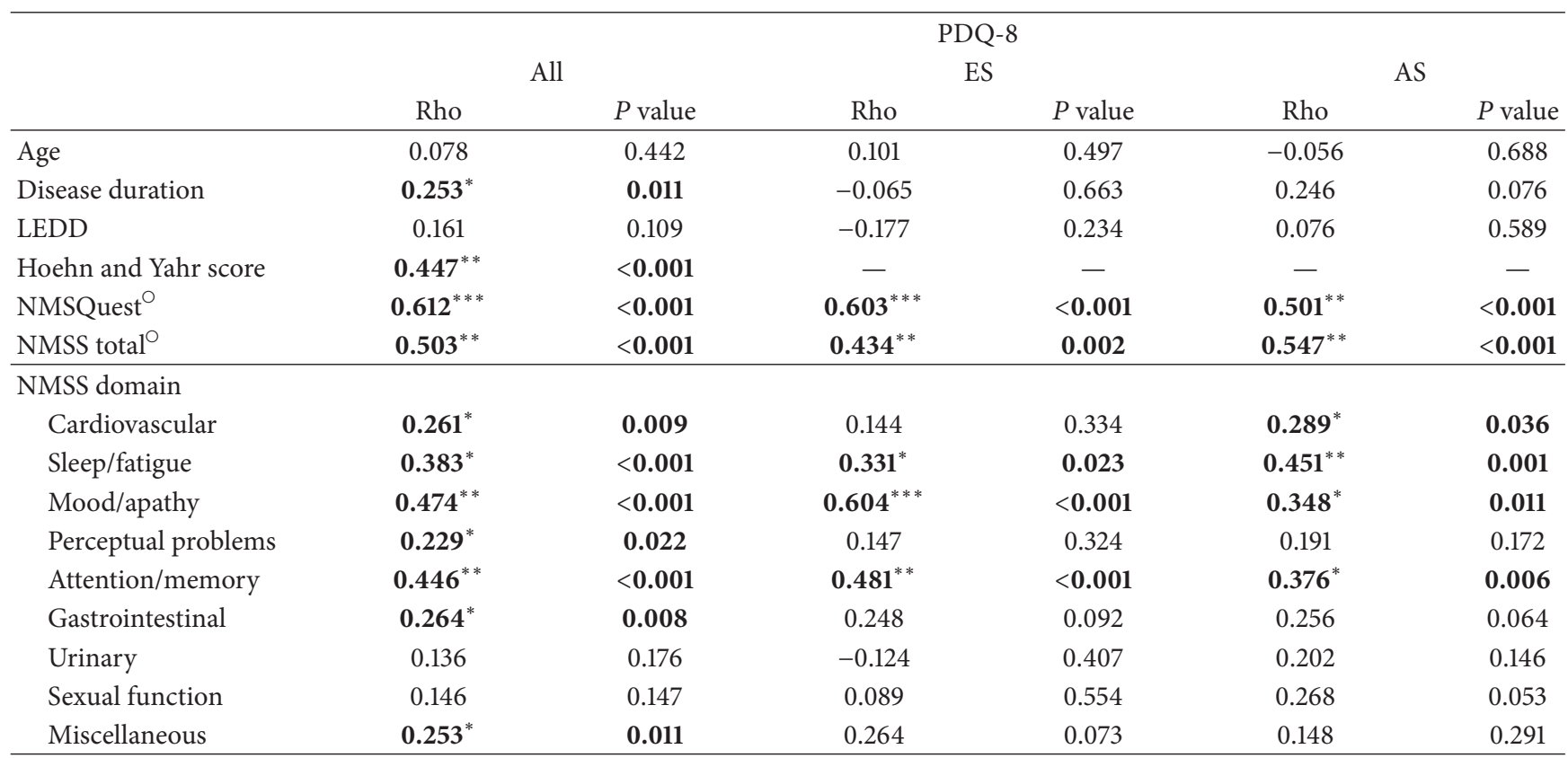

Abbreviations: AS: advanced-stage Parkinson's disease; ES: early-stage Parkinson’s disease; LEDD: levodopa equivalent daily dose.

${ }^{\circ}$ For explanation, see Section 2.2.

The strength of the association for correlation coefficients is interpreted as follows: ${ }^{*} 0.20$ to 0.39 , weak; ${ }^{* *} 0.40$ to 0.59 , moderate; ${ }^{* * *} 0.60$ to 0.79 , strong; significant values are bold.

not reveal any fundamental differences; however, the degree of the association with HRQoL differed. The neuropsychiatric domains attention/memory and mood/apathy correlated moderately to strongly with HRQoL in the ES group, while HRQoL was moderately related to the sleep/fatigue domain in the AS group. Other weak but still significantly correlating domains were sleep/fatigue in ES and cardiovascular aspects, attention/memory, and mood/apathy in AS. Our data are thus in line with previous studies reporting that fatigue, sleep disturbances, apathy, and mood are independent determinants of HRQoL [3-5, 19-22].

As our subjects had been optimally "tuned up" with dopaminergic therapy (according to the clinicians' impressions) and fluctuating patients were assessed in their optimal "on" state, we can assume that nondopaminergic lesions influenced their HRQoL the most.

An important limitation of our study is that the most advanced phases of disease were underrepresented. Other drawbacks are the absence of severity measures for motor symptoms, like UPDRS motor score, to determine the impact of motor system involvement, and the fact that special attention was not given to nondopaminergic add-on therapies, for example, antidepressants, hypnotics, cognitives, and laxatives.

In conclusion, our data confirm that NMS are very common and significantly influence HRQoL even in PD 
patients on dopaminergic therapy. These observations indicate that systematic, active screening for NMS and above all therapeutic strategies for neuropsychiatric disorders (mood, cognition, sleep, and fatigue) are urgently required both at the initial diagnosis of PD and across the entire disease course. Further studies are needed to investigate how to improve the HRQoL of PD patients and relieve their NMS.

\section{Conflict of Interests}

The authors declare that there is no conflict of interests regarding the publication of this paper.

\section{Authors' Contribution}

Peter Valkovic and Jan Harsany participated equally in this work.

\section{Acknowledgment}

The authors wish to thank Judy Benson for copyediting the paper.

\section{References}

[1] M. Drobný and E. Kurča, "Possible extrapyramidal system degradation in Parkinson's disease," Brain Research Bulletin, vol. 53, no. 4, pp. 425-430, 2000.

[2] K. R. Chaudhuri, J. M. Rojo, A. H. Schapira et al., "A proposal for a comprehensive grading of Parkinson's disease severity combining motor and non-motor assessments: meeting an unmet need," PLoS ONE, vol. 8, Article ID e57221, 2013.

[3] P. Martinez-Martin, C. Rodriguez-Blazquez, M. M. Kurtis, and K. R. Chaudhuri, "The impact of non-motor symptoms on health-related quality of life of patients with Parkinson's disease," Movement Disorders, vol. 26, no. 3, pp. 399-406, 2011.

[4] S.-E. Soh, M. E. Morris, and J. L. McGinley, "Determinants of health-related quality of life in Parkinson's disease: a systematic review," Parkinsonism \& Related Disorders, vol. 17, no. 1, pp. 1-9, 2011.

[5] D. A. Gallagher, A. J. Lees, and A. Schrag, "What are the most important nonmotor symptoms in patients with Parkinson's disease and are we missing them?" Movement Disorders, vol. 25, no. 15, pp. 2493-2500, 2010.

[6] J. Shearer, C. Green, C. E. Counsell, and J. P. Zajicek, “The impact of motor and non motor symptoms on health state values in newly diagnosed idiopathic Parkinson's disease," Journal of Neurology, vol. 259, no. 3, pp. 462-468, 2012.

[7] A. M. Bonnet, M. F. Jutras, V. Czernecki, J. C. Corvol, and M. Vidailhet, "Nonmotor symptoms in Parkinson's disease in 2012: relevant clinical aspects," Parkinson's Disease, vol. 2012, Article ID 198316, 15 pages, 2012.

[8] K. R. Chaudhuri and A. H. Schapira, "Non-motor symptoms of Parkinson's disease: dopaminergic pathophysiology and treatment," The Lancet Neurology, vol. 8, no. 5, pp. 464-474, 2009.

[9] J. E. Ahlskog, "Beating a dead horse: dopamine and Parkinson disease," Neurology, vol. 69, no. 17, pp. 1701-1711, 2007.

[10] A. Storch, C. B. Schneider, M. Wolz et al., "Nonmotor fluctuations in Parkinson disease: severity and correlation with motor complications," Neurology, vol. 80, no. 9, pp. 800-809, 2013.
[11] A. J. Hughes, S. E. Daniel, S. Blankson, and A. J. Lees, "A clinicopathologic study of 100 cases of Parkinson's disease," Archives of Neurology, vol. 50, no. 2, pp. 140-148, 1993.

[12] J. Jankovic, M. McDermott, J. Carter et al., "Variable expression of Parkinson's disease: a base-line analysis of the DATATOP cohort," Neurology, vol. 40, no. 10, pp. 1529-1534, 1990.

[13] C. L. Tomlinson, R. Stowe, S. Patel, C. Rick, R. Gray, and C. E. Clarke, "Systematic review of levodopa dose equivalency reporting in Parkinson's disease," Movement Disorders, vol. 25, no. 15, pp. 2649-2653, 2010.

[14] C. Jenkinson, R. Fitzpatrick, V. Peto, R. Greenhall, and N. Hyman, "The PDQ-8: development and validation of a shortform Parkinson's disease questionnaire," Psychology \& Health, vol. 12, no. 6, pp. 805-814, 1997.

[15] K. R. Chaudhuri, P. Martinez-Martin, A. H. Schapira et al., "International multicenter pilot study of the first comprehensive self-completed nonmotor symptoms questionnaire for Parkinson's disease: the NMSQuest study," Movement Disorders, vol. 21, no. 7, pp. 916-923, 2006.

[16] K. R. Chaudhuri, P. Martinez-Martin, R. G. Brown et al., "The metric properties of a novel non-motor symptoms scale for Parkinson's disease: results from an international pilot study," Movement Disorders, vol. 22, no. 13, pp. 1901-1911, 2007.

[17] T. D. V. Swinscow and M. J. Campbell, Statistics at Square One, BMJ, London, UK, 9th edition, 1997.

[18] D. Crosiers, B. Pickut, J. Theuns et al., "Non-motor symptoms in a Flanders-Belgian population of 215 Parkinson's disease patients as assessed by the Non-Motor Symptoms Questionnaire," American Journal of Neurodegenerative Disease, vol. 1, no. 2, pp. 160-167, 2012.

[19] P. Barone, A. Antonini, C. Colosimo et al., "The PRIAMO study: a multicenter assessment of nonmotor symptoms and their impact on quality of life in Parkinson's disease," Movement Disorders, vol. 24, no. 11, pp. 1641-1649, 2009.

[20] M. Skorvanek, J. Rosenberger, Z. Gdovinova et al., "Apathy in elderly nondemented patients with Parkinson's disease: clinical determinants and relationship to quality of life," Journal of Geriatric Psychiatry and Neurology, vol. 26, no. 4, pp. 237-243, 2013.

[21] C. Hinnell, C. S. Hurt, S. Landau et al., "Nonmotor versus motor symptoms: how much do they matter to health status in Parkinson's disease?" Movement Disorders, vol. 27, no. 2, pp. 236-241, 2012.

[22] E. Havlikova, J. P. van Dijk, I. Nagyova et al., "The impact of sleep and mood disorders on quality of life in Parkinson's disease patients," Journal of Neurology, vol. 258, no. 12, pp. 22222229, 2011. 


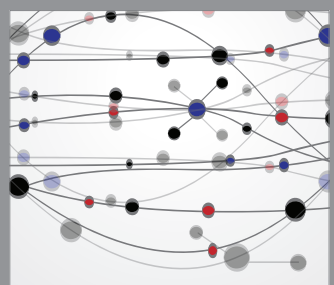

The Scientific World Journal
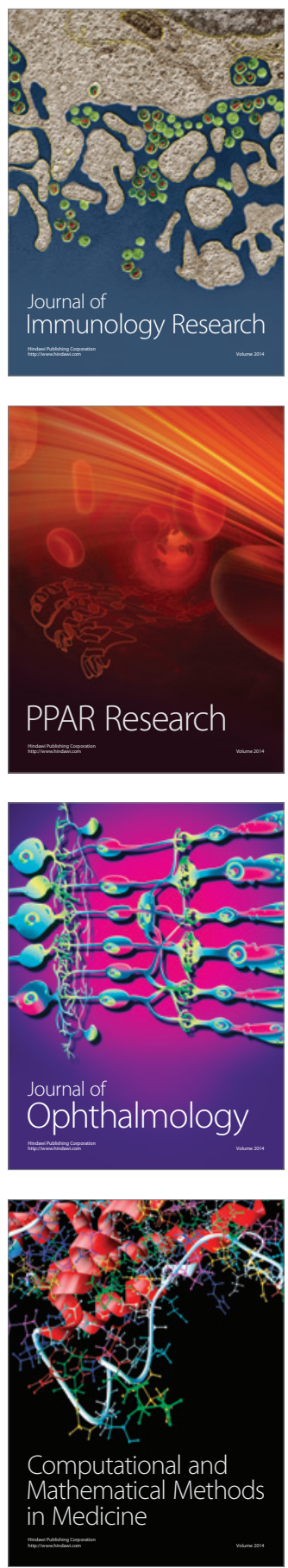

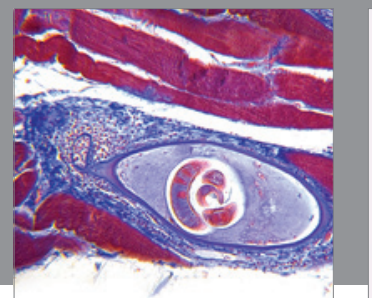

Gastroenterology

Research and Practice
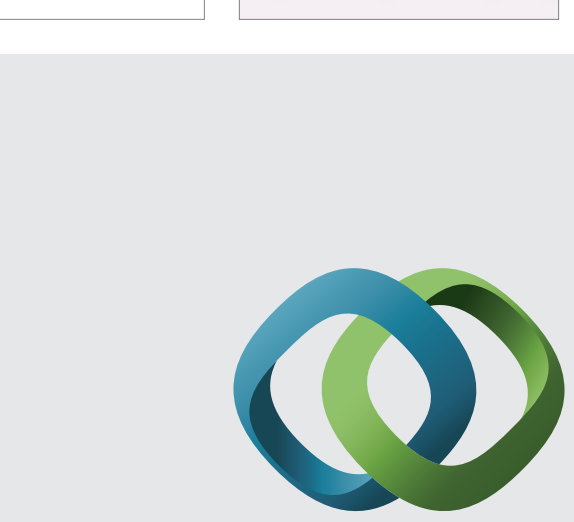

\section{Hindawi}

Submit your manuscripts at

http://www.hindawi.com
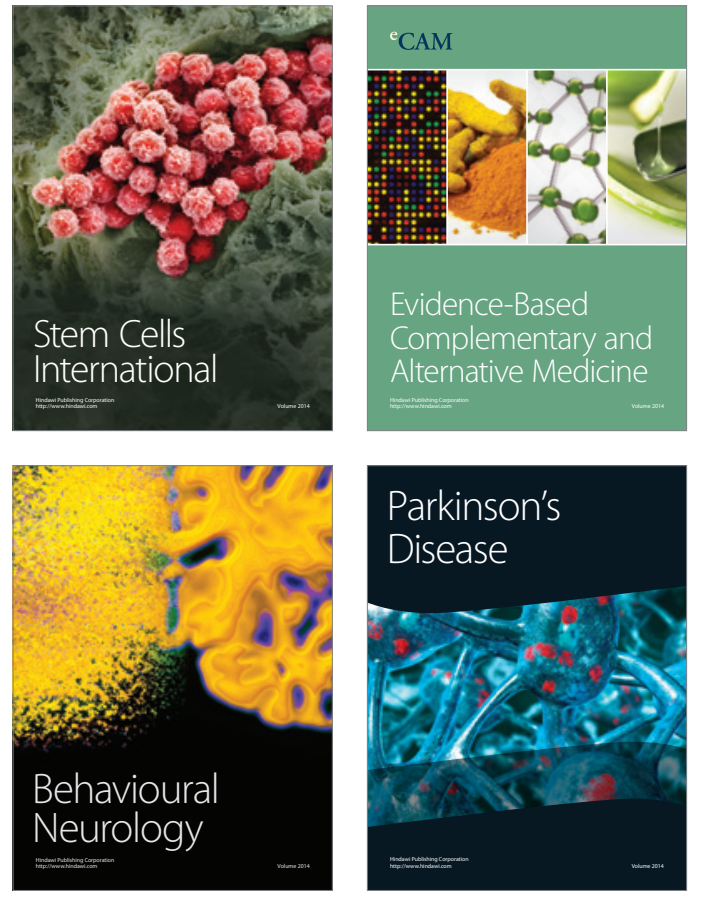
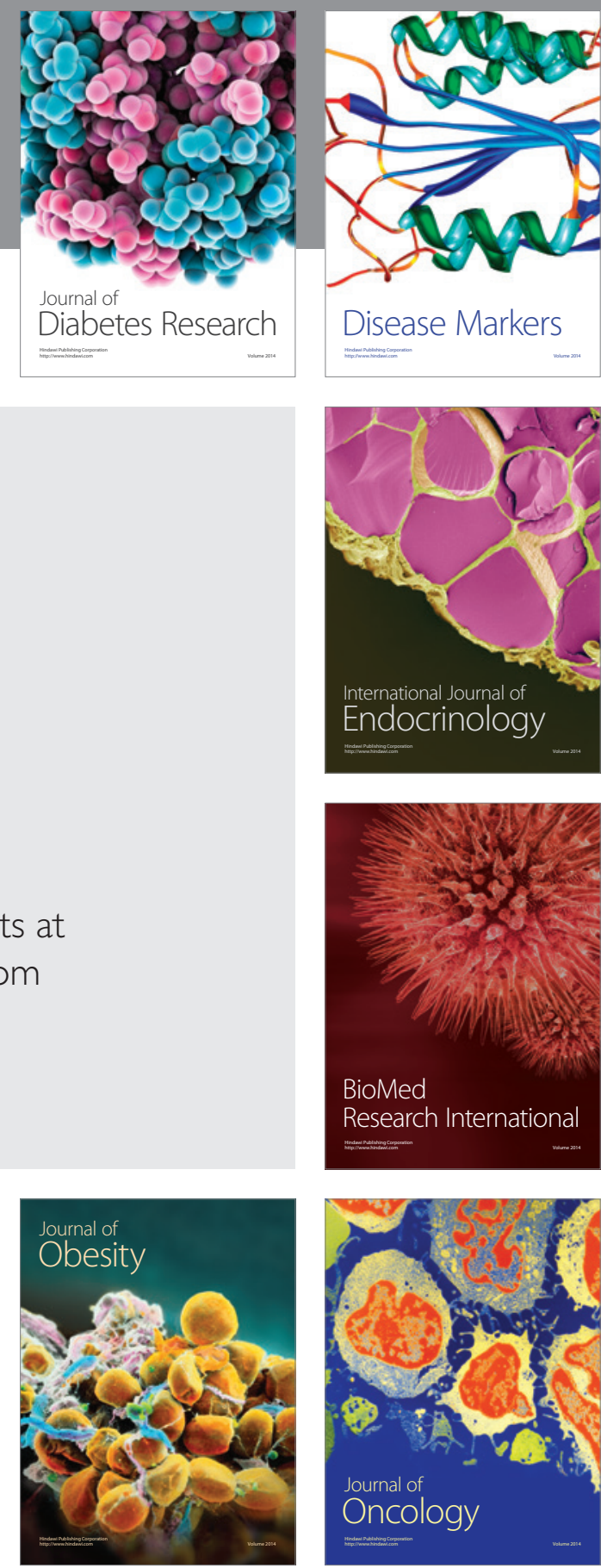

Disease Markers
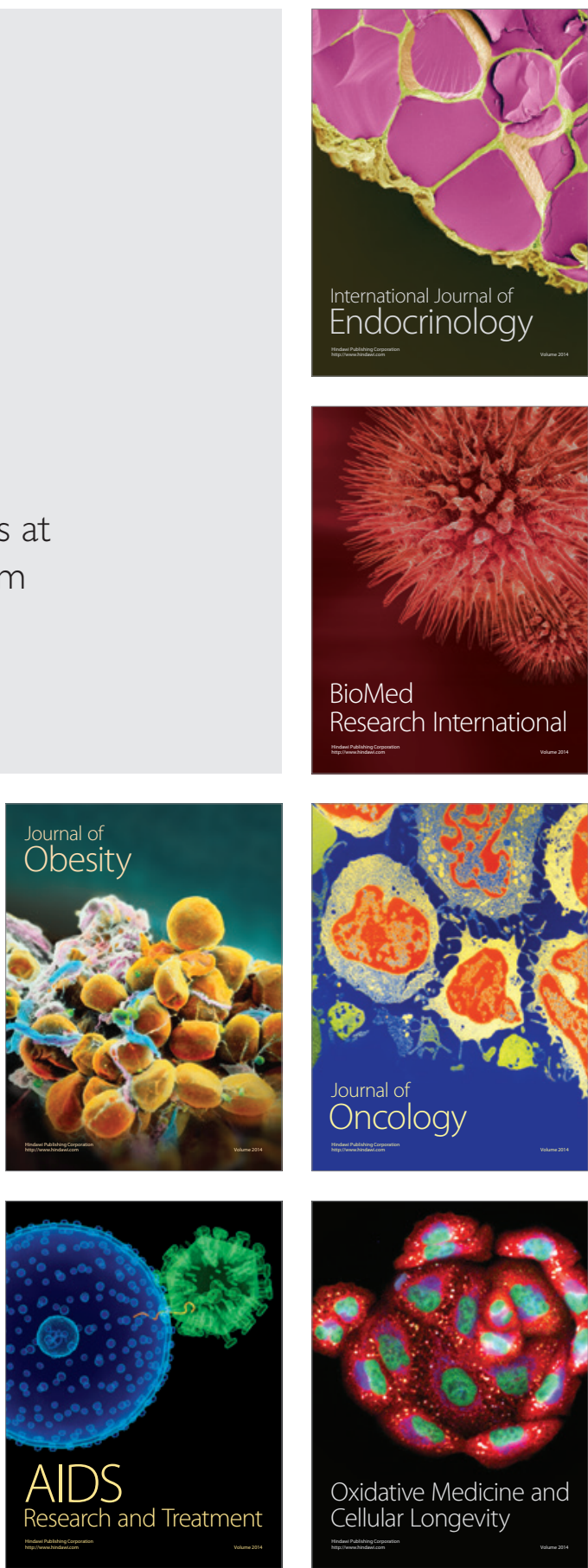